Supporting Information

\title{
Electron Paramagnetic Resonance Spectroscopic Identification of the Fe-S Clusters in the SPASM Domain-Containing Radical SAM Enzyme PqqE
}

\author{
Lizhi Tao ${ }^{1 \#}$, Wen Zhu ${ }^{2 \#}$, Judith P. Klinman ${ }^{2 *}$ and R. David Britt ${ }^{1 *}$
}

${ }^{1}$ Department of Chemistry, University of California, Davis, California 95616, United States

${ }^{2}$ Department of Chemistry, Department of Molecular and Cell Biology, and California Institute for Quantitative Biosciences, University of California, Berkeley, California 94720, United States

*Corresponding Authors: klinman@berkeley.edu and rdbritt@ucdavis.edu 


\section{Table of Contents}

Table S1. Primers and DNA templates used in the mutagenesis in this study.

Figure S1. Temperature dependence and power dependence of the EPR signals of dithionitereduced $R S$-only variant.

Figure S2. Temperature dependence and power dependence of the EPR signals of dithionitereduced AuxI/AuxII.

Figure S3. X-band (9.37 GHz) CW EPR and Q-band (34.0 GHz) pseudo-modulated electron spin-echo detected field-swept EPR spectra of dithionite-reduced AuxI/AuxII sample with the addition of $\sim 100$ equivalents of $\mathrm{K}^{13} \mathrm{C}^{15} \mathrm{~N}$.

Figure S4. X-band (9.37 GHz) CW EPR and Q-band (34.0 GHz) pseudo-modulated electron spin-echo detected field-swept EPR spectra of dithionite-reduced AuxI/AuxII/D319H and AuxI/AuxII/D319C.

Figure S5. The simulated X-band $(9.403 \mathrm{GHz}){ }^{14} \mathrm{~N}-\mathrm{HYSOCRE}$ spectrum of dithionitereduced AuxI/AuxII/D319H.

Figure S6. X-band (9.37 GHz) CW EPR spectra of Ti(III) citrate-reduced PqqE samples of WT, AuxI/AuxII, RS/AuxI, RS/AuxII, AuxI/AuxIID319H, AuxI/AuxII/D319C and RS-only.

Figure S7. X-band (9.37 GHz) CW EPR spectra of Ti(III) citrate-reduced RS/AuxI PqqE variant before and after the addition of $\sim 110$ equivalents of $\mathrm{K}^{13} \mathrm{C}^{15} \mathrm{~N}$.

Figure S8. Q-band (34.0 GHz) pseudo-modulated electron spin-echo detected field-swept EPR spectrum of Ti(III) citrate-reduced WT PqqE.

Figure S9. X-band $(9.37 \mathrm{GHz}) \mathrm{CW}$ EPR spectra of dithionite-reduced RS/AuxI and RS/AuxII.

Figure S10. Temperature dependence and power dependence of the EPR signals of Ti(III) citrate-reduced WT PqqE.

Figure S11. X-band (9.37 GHz) CW EPR spectra of Eu(II)-DTPA-reduced WT PqqE before and after desalting.

Figure S12. X-band $(9.37 \mathrm{GHz}) \mathrm{CW}$ EPR of dithionite-reduced two batches of the AuxI/AuxII samples.

Figure S13. Temperature dependence and power dependence of the EPR signals of [2Fe-2S] cluster at $10 \mathrm{~K}$. 
Table S1 Primers and DNA templates used in the mutagenesis in this study.

\begin{tabular}{|c|c|c|}
\hline Primer & Sequence & DNA template \\
\hline C28A forward & $\begin{array}{l}\text { 5'- GTT AAC GCA CCG TGC } \\
\text { TCC ATT GCG TTG C -3' }\end{array}$ & \multirow{2}{*}{ wild-type } \\
\hline C28A reverse & $\begin{array}{c}\text { 5'- GCA ACG CAA TGG AGC } \\
\text { ACG GTG CGT TAA C -3' }\end{array}$ & \\
\hline $\mathrm{C} 28 \mathrm{~A} / \mathrm{C} 32 \mathrm{~A} / \mathrm{C} 35 \mathrm{~A}$ forward & $\begin{array}{c}\text { 5'- CAC CGT GCT CCA TTG } \\
\text { CGT GCC CCT TAT GCT AGT } \\
\text { AAT C - } 3^{\prime}\end{array}$ & \multirow{2}{*}{$\mathrm{C} 28 \mathrm{~A}$} \\
\hline $\mathrm{C} 28 \mathrm{~A} / \mathrm{C} 32 \mathrm{~A} / \mathrm{C} 35 \mathrm{~A}$ reverse & $\begin{array}{l}\text { 5'- GAT TAC TAG CAT AAG } \\
\text { GGG CAC GCA ATG GAG CAC } \\
\text { GGT G -3' }\end{array}$ & \\
\hline C28A/C32A/C35A/D319H forward & $\begin{array}{l}\text { 5'- GAT CGT CGT GAG AAA } \\
\text { CAT TGG GGG GGA TGT C -3' }\end{array}$ & \multirow{2}{*}{$\mathrm{C} 28 \mathrm{~A} / \mathrm{C} 32 \mathrm{~A} / \mathrm{C} 35 \mathrm{~A}$} \\
\hline C28A/C32A/C35A/D319H reverse & $\begin{array}{l}\text { 5'- GAC ATC CCC CCC AAT } \\
\text { GTT TCT CAC GAC GAT C -3' }\end{array}$ & \\
\hline C28A/C32A/C35A/D319C forward & $\begin{array}{l}\text { 5'- GAT CGT CGT GAG AAA } \\
\text { TGT TGG GGG GGA TGT C -3' }\end{array}$ & \multirow{2}{*}{$\mathrm{C} 28 \mathrm{~A} / \mathrm{C} 32 \mathrm{~A} / \mathrm{C} 35 \mathrm{~A}$} \\
\hline $\mathrm{C} 28 \mathrm{~A} / \mathrm{C} 32 \mathrm{~A} / \mathrm{C} 35 \mathrm{~A} / \mathrm{D} 319 \mathrm{C}$ reverse & $\begin{array}{l}\text { 5'- GAC ATC CCC CCC AAC } \\
\text { ATT TCT CAC GAC GAT C -3' }\end{array}$ & \\
\hline C310A forward & $\begin{array}{l}\text { 5'- GAT GAA GGA GCC AGC } \\
\text { TCG CTC CTG TGA TCG -3' }\end{array}$ & \multirow{2}{*}{ wild-type } \\
\hline C310A reverse & $\begin{array}{c}\text { 5'- CGA TCA CAG GAG CGA } \\
\text { GCT GGC TCC TTC ATC -3' }\end{array}$ & \\
\hline C310A/C313A forward & $\begin{array}{c}\text { 5'- GCC AGC TCG CTC CGC } \\
\text { TGA TCG TCG TGA G -3' }\end{array}$ & \multirow{2}{*}{ C310A } \\
\hline C310A/C313A reverse & $\begin{array}{c}\text { 5'- CTC ACG ACG ATC AGC } \\
\text { GGA GCG AGC TGG C -3' }\end{array}$ & \\
\hline C268A forward & $\begin{array}{l}\text { 5'- GGA AAG TCT TAC CTG } \\
\text { CCC ACG CTG CTG AGA C -3' }\end{array}$ & \multirow{2}{*}{ wild-type } \\
\hline C268A reverse & $\begin{array}{l}\text { 5'- GTC TCA GCA GCG TGG } \\
\text { GCA GGT AAG ACT TTC C -3' }\end{array}$ & \\
\hline C248A/C268A forward & $\begin{array}{l}\text { 5'- CAA ATA TCC TAA GGC } \\
\text { GTG GCC GGG CGG TTG G -3' }\end{array}$ & \multirow{2}{*}{$\mathrm{C} 268 \mathrm{~A}$} \\
\hline C248A/C268A reverse & $\begin{array}{l}\text { 5'- CCA ACC GCC CGG CCA } \\
\text { CGC CTT AGG ATA TTT G -3' }\end{array}$ & \\
\hline C310A/C313A/C323A forward & $\begin{array}{c}\text { 5'- GAT TGG GGG GGA GCT } \\
\text { CGC TGT CAA G -3' }\end{array}$ & \multirow{2}{*}{ C310A/C313A } \\
\hline $\mathrm{C} 310 \mathrm{~A} / \mathrm{C} 313 \mathrm{~A} / \mathrm{C} 323 \mathrm{~A}$ reverse & $\begin{array}{c}\text { 5'- CTT GAC AGC GAG CTC } \\
\text { CCC CCC AAT C -3' }\end{array}$ & \\
\hline $\mathrm{C} 310 \mathrm{~A} / \mathrm{C} 313 \mathrm{~A} / \mathrm{C} 323 \mathrm{~A} / \mathrm{C} 325 \mathrm{~A}$ forward & $\begin{array}{l}\text { 5'- GGG AGC TCG CGC TCA } \\
\text { AGC ATT GGC CTT AAC -3' }\end{array}$ & \multirow{2}{*}{$\mathrm{C} 310 \mathrm{~A} / \mathrm{C} 313 \mathrm{~A} / \mathrm{C} 323 \mathrm{~A}$} \\
\hline $\mathrm{C} 310 \mathrm{~A} / \mathrm{C} 313 \mathrm{~A} / \mathrm{C} 323 \mathrm{~A} / \mathrm{C} 325 \mathrm{~A}$ reverse & $\begin{array}{l}\text { 5'- GTT AAG GCC AAT GCT } \\
\text { TGA GCG CGA GCT CCC -3' }\end{array}$ & \\
\hline
\end{tabular}



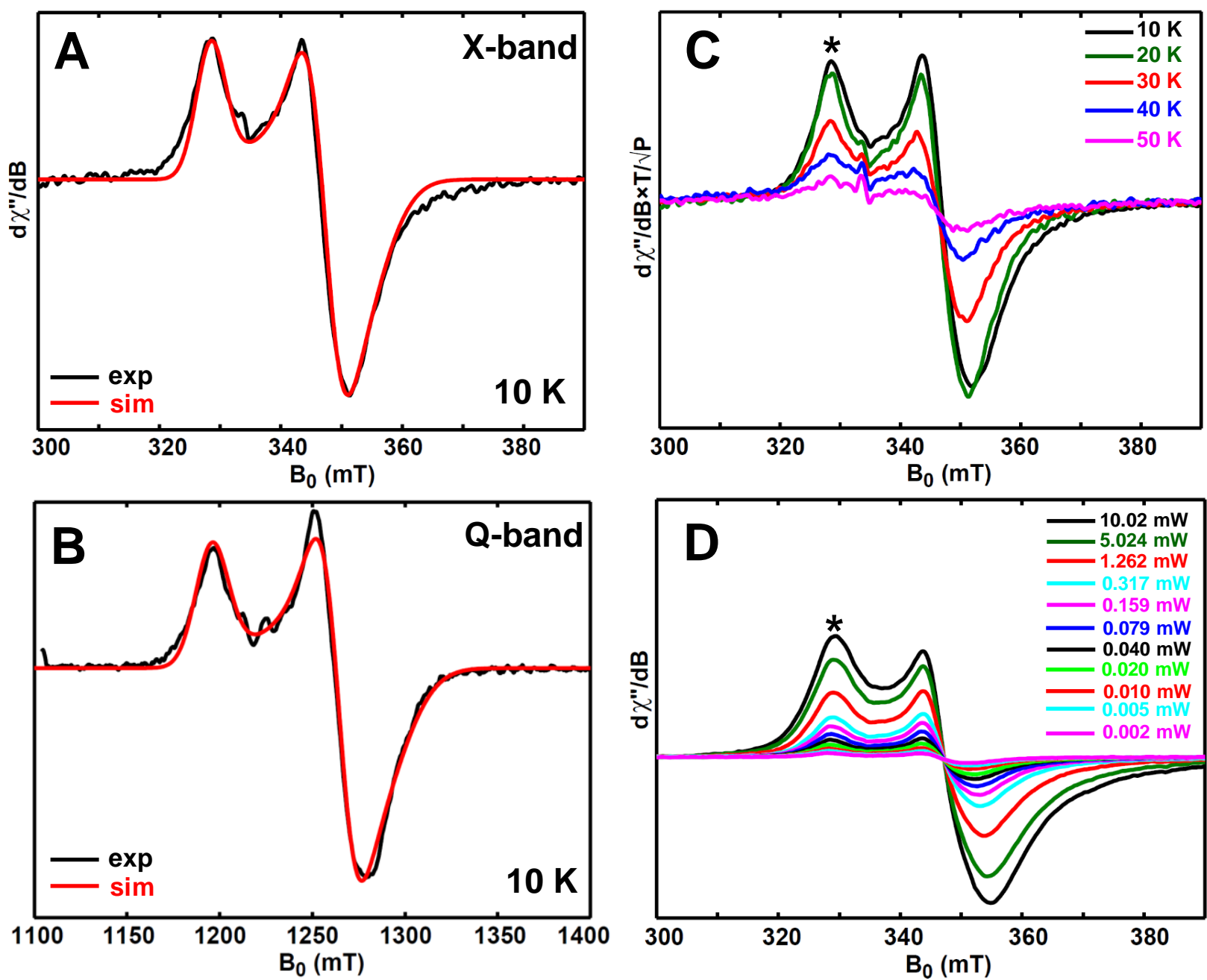

Figure S1. X-band (9.38 GHz) CW EPR (A) and Q-band (34.0 GHz) pseudo-modulated electron spin-echo detected field-swept EPR spectra (B) of dithionite-reduced double-knockout PqqE variant of $R S$ only. The black traces are experimental spectra, while the red traces are the simulated spectra by employing the $g$-values $=[2.040,1.927,1.897]$. The X-band CW EPR spectra was recorded at $10 \mathrm{~K}$, with $0.02 \mathrm{~mW}$ microwave power (no saturation). The Q-band EPR spectra was recorded at $10 \mathrm{~K}$ by using a two-pulse sequence of $\pi / 2-\tau-\pi$ - $\tau$-echo, with $\pi / 2=12 \mathrm{~ns}$ and $\tau=300 \mathrm{~ns}$. The modulation amplitude of $3.0 \mathrm{mT}$ was used to convert the absorption spectra to the pseudo-modulated spectra in (B).

Temperature dependence (C) and power dependence (D) of the EPR signals of dithionitereduced $R S$-only variant. The asterisk indicates the $g_{1} 2.040$ position, where the peak amplitudes are employed as the signal intensities of the RS cluster shown in Figure 4 (blue diamonds). 

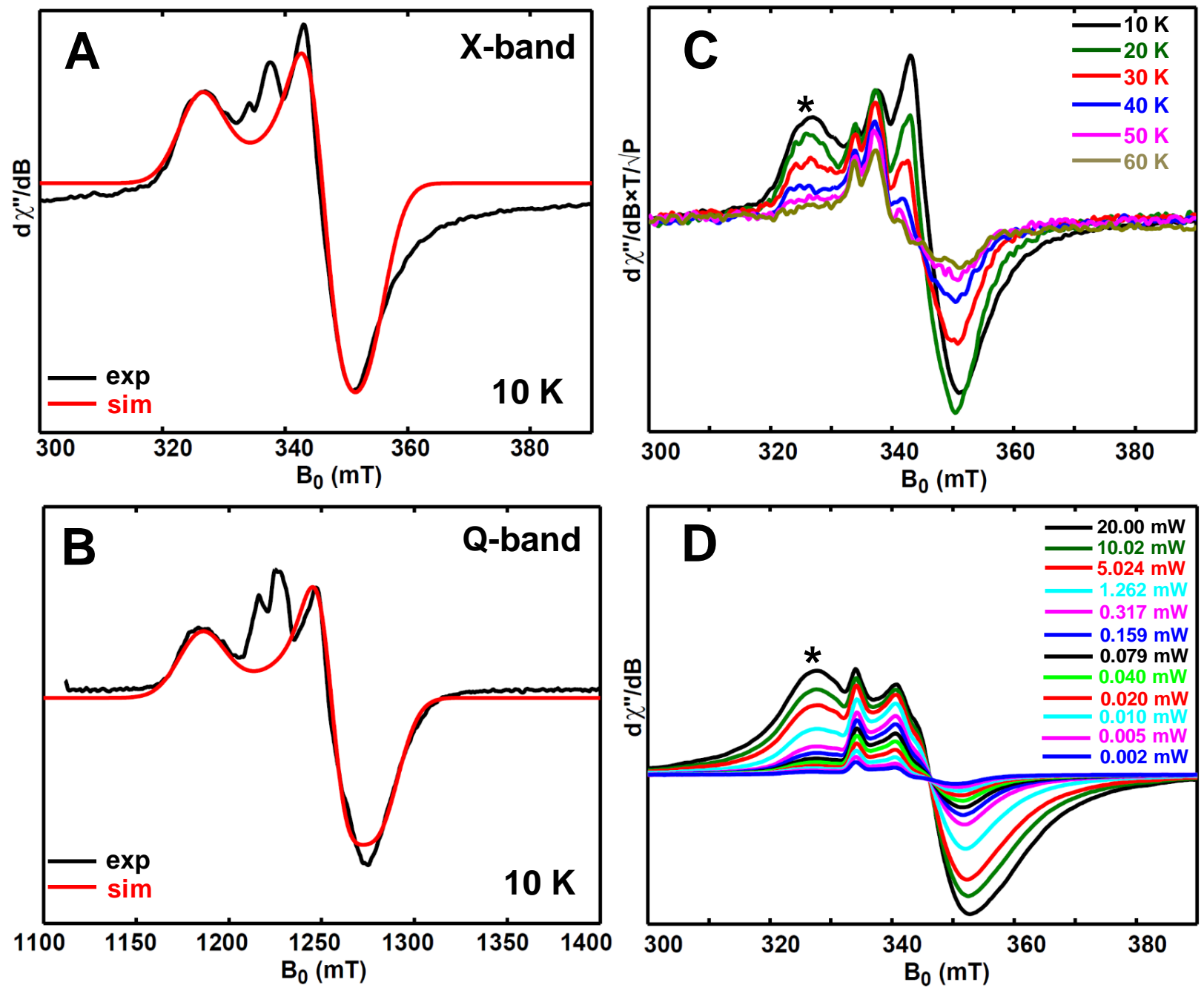

Figure S2. X-band (9.38 GHz) CW EPR (A) and Q-band (34.0 GHz) pseudo-modulated electron spin-echo detected field-swept EPR spectra (B) of dithionite-reduced AuxI/AuxII. The black traces are experimental spectra, while the red traces are the simulated spectra by employing the $g$-values $=[2.059,1.940,1.903]$. The X-band CW EPR spectrum was recorded at $10 \mathrm{~K}$, with $0.02 \mathrm{~mW}$ microwave power (no saturation). The Q-band EPR spectrum was recorded at $10 \mathrm{~K}$ by using a two-pulse sequence of $\pi / 2-\tau$ - $\pi$ - $\tau$-echo, with $\pi / 2=12$ ns and $\tau=300$ ns. The modulation amplitude of $3.0 \mathrm{mT}$ was used to convert the absorption spectra to the pseudo-modulated spectra in (B).

Temperature dependence (C) and power dependence (D) of the EPR signals of dithionitereduced AuxI/AuxII. The asterisk indicates the $g_{1} 2.059$ position, where the peak amplitudes are employed as the major-component signal intensities of the AuxII cluster shown in Figure 4 (magenta squares). 

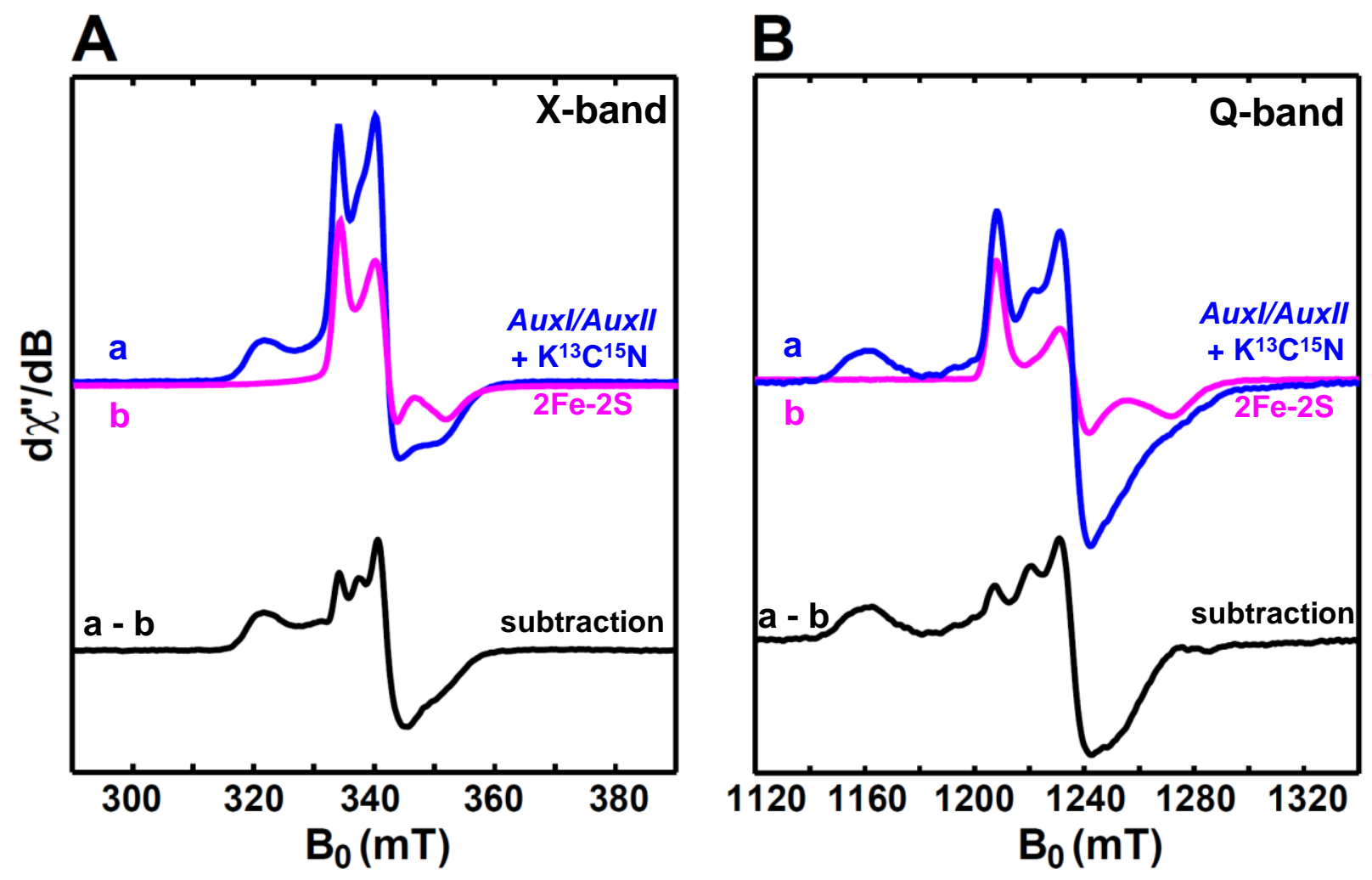

Figure S3. X-band (9.37 GHz) CW EPR (A) and Q-band (34.0 GHz) pseudo-modulated electron spin-echo detected field-swept EPR spectra (B) of dithionite-reduced AuxI/AuxII sample with the addition of $\sim 100$ equivalents of $\mathrm{K}^{13} \mathrm{C}^{15} \mathrm{~N}$. The blue traces are the original spectra, showing that the $[2 \mathrm{Fe}-2 \mathrm{~S}]^{+}$cluster signal was observed upon the $\mathrm{K}^{13} \mathrm{C}^{15} \mathrm{~N}$ addition. The black traces are the spectra we show in Figure 6 , with the $[2 \mathrm{Fe}-2 \mathrm{~S}]^{+}$signal being subtracted away. The $[2 \mathrm{Fe}-2 \mathrm{~S}]^{+}$ cluster signal (magenta traces) is adopted from the high-temperature EPR spectra of dithionitereduced RS/AuxI (Figure S13), where only the $[2 \mathrm{Fe}-2 \mathrm{~S}]^{+}$cluster signal is persisting due to its slow-relaxation properties. 

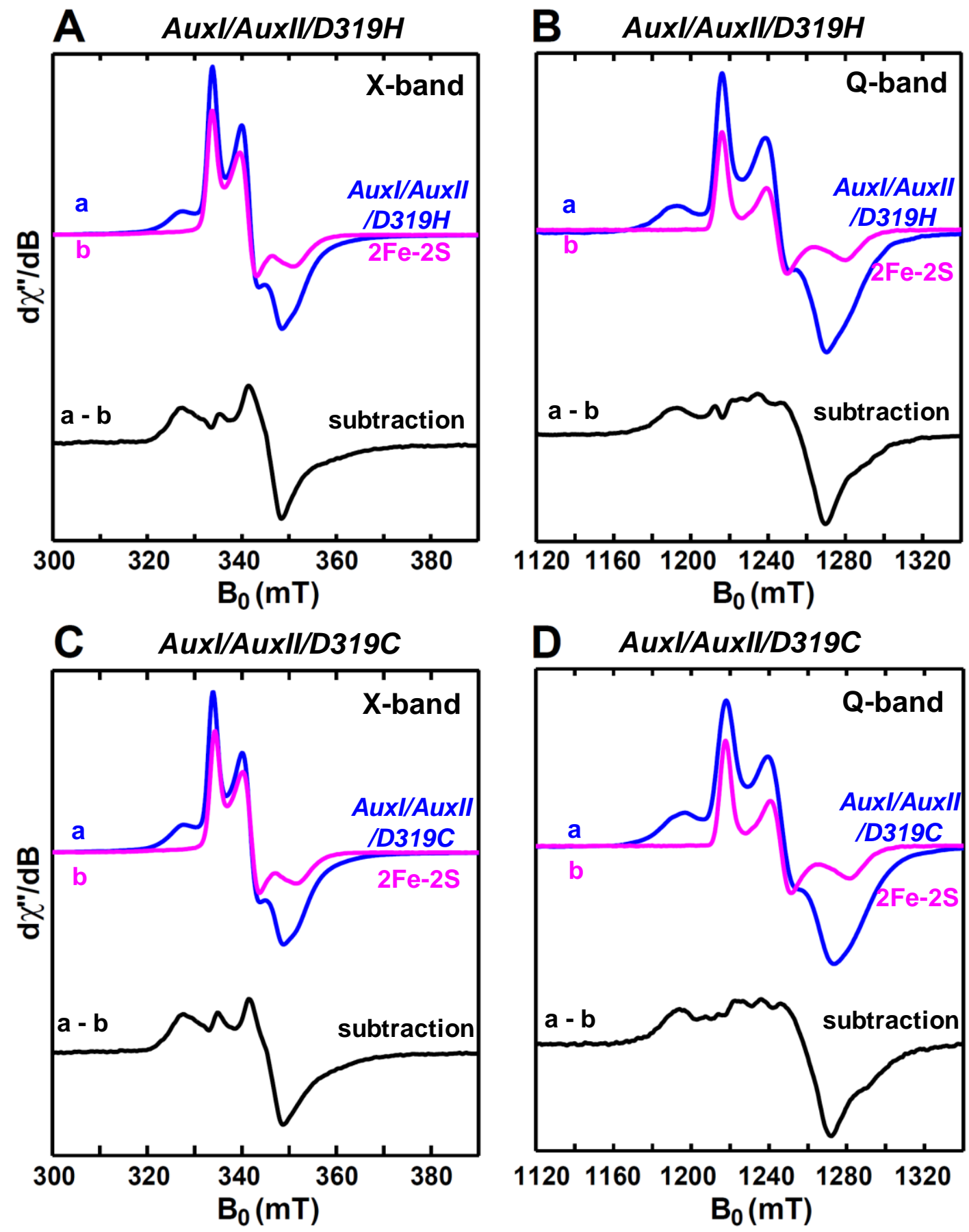

Figure S4. X-band (9.37 GHz) CW EPR (A, C) and Q-band (34.0 GHz) pseudo-modulated electron spin-echo detected field-swept EPR spectra (B, D) of dithionite-reduced AuxI/AuxII/D319H and AuxI/AuxII/D319C. The blue traces are the original spectra, showing that the $[2 \mathrm{Fe}-2 \mathrm{~S}]^{+}$cluster signal was observed. The black traces are the spectra we show in Figure 6 , with the $[2 \mathrm{Fe}-2 \mathrm{~S}]^{+}$signal being subtracted away. 

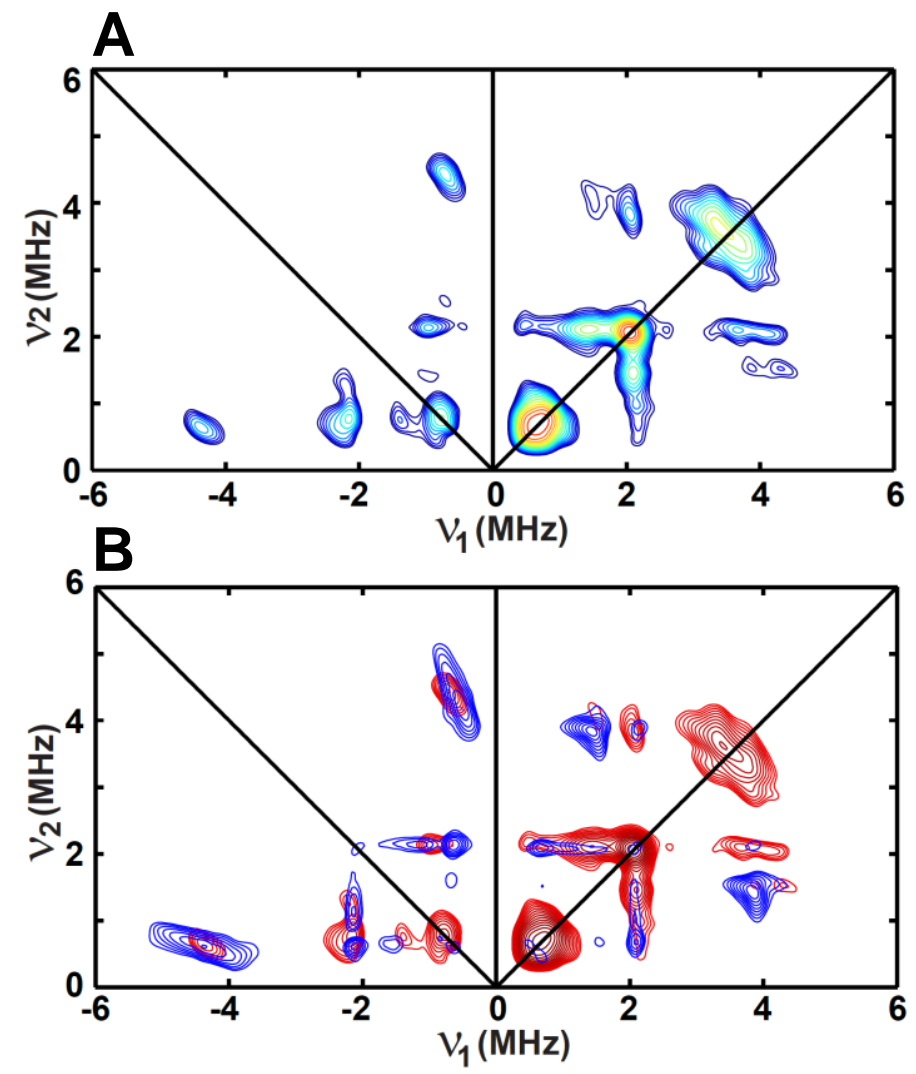

Figure S5. (A) X-band $(9.403 \mathrm{GHz})$ HYSCORE spectra of dithionite-reduced AuxI/AuxII/D319H acquired at the magnetic field position $(328.4 \mathrm{mT})$ corresponding to the $g$ value of 2.046. This spectrum is also shown in Figure 8C.

(B) The simulated ${ }^{14} \mathrm{~N}-\mathrm{HYSOCRE}$ spectrum are shown in blue (contour plot) by using the parameters of $g=[2.087,1.955,1.941] ; A\left({ }^{14} \mathrm{~N}\right)=[0.73,3.25,1.01 \mathrm{MHz}$, Euler angle $=[55,100$, $25]^{\circ}$ referring to $\mathbf{A}$ tensor to $\mathbf{g}$ tensor, $P\left({ }^{14} \mathrm{~N}\right)=[0.23,-1.02,0.79] \mathrm{MHz}$, Euler angle $=[50,27$, $20]^{\circ}$ referring to $\mathbf{P}$ tensor to $\mathbf{A}$ tensor. The quadrupole coupling values we report here are defined as $\left[P_{1}, P_{2}, P_{3}\right]=e^{2} Q q / 4 I(2 I-1) h[-1+\eta, 2,-1-\eta]$, with the asymmetry parameter $\eta=0.55$. 

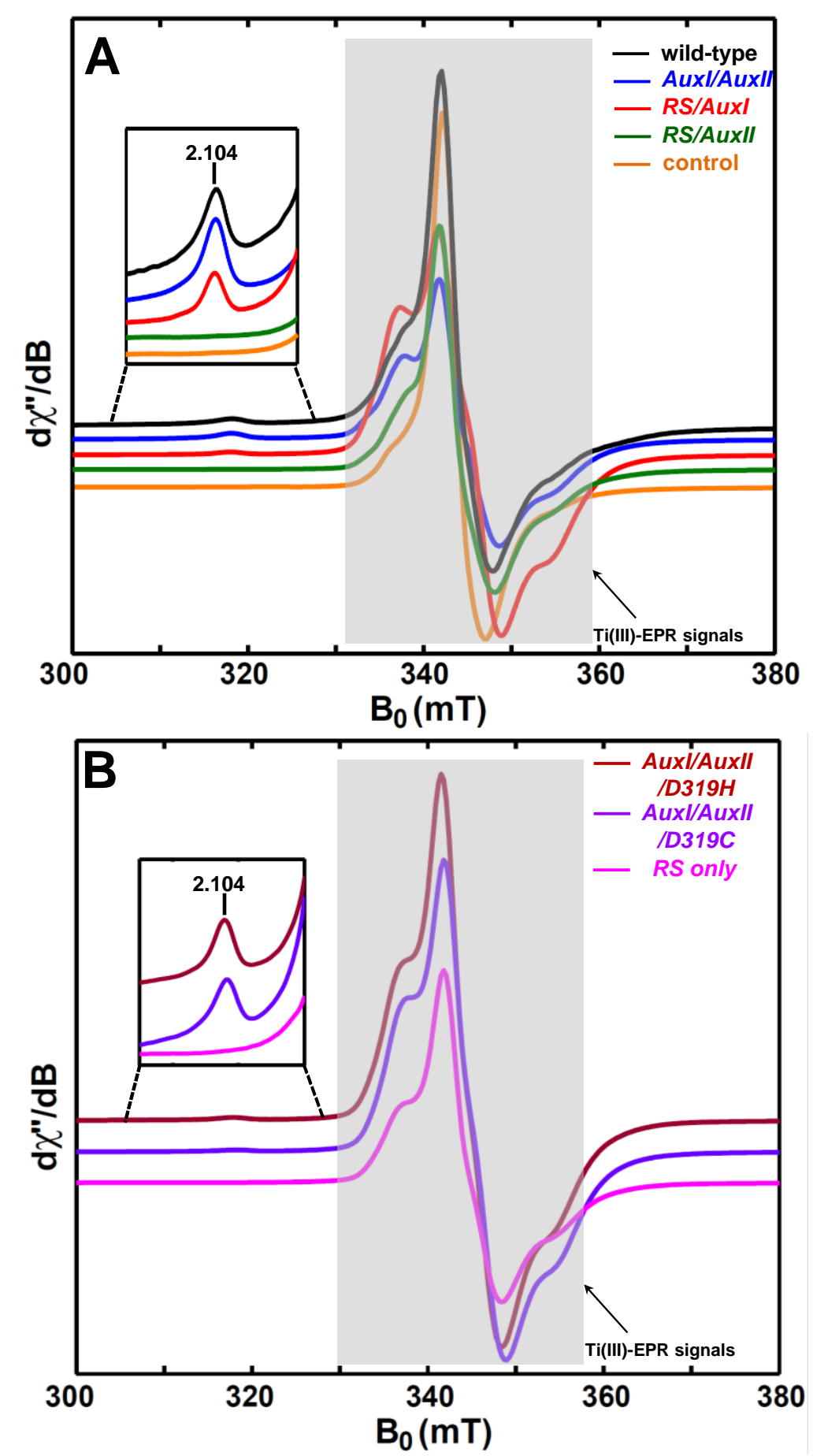

Figure S6. X-band (9.37 GHz) CW EPR spectra of Ti(III) citrate-reduced PqqE samples of wildtype (black trace), AuxI/AuxII (blue trace), RS/AuxI (red trace), RS/AuxII (green trace), AuxI/AuxII/D319H (burgundy trace), AuxI/AuxII/D319C (purple trace) and RS only (magenta trace). The control sample (orange trace) is Ti(III) citrate in HEPES-buffered solution. The CW EPR spectra were recorded at $10 \mathrm{~K}$ using $2.518 \mathrm{~mW}$ microwave power (no saturation). 


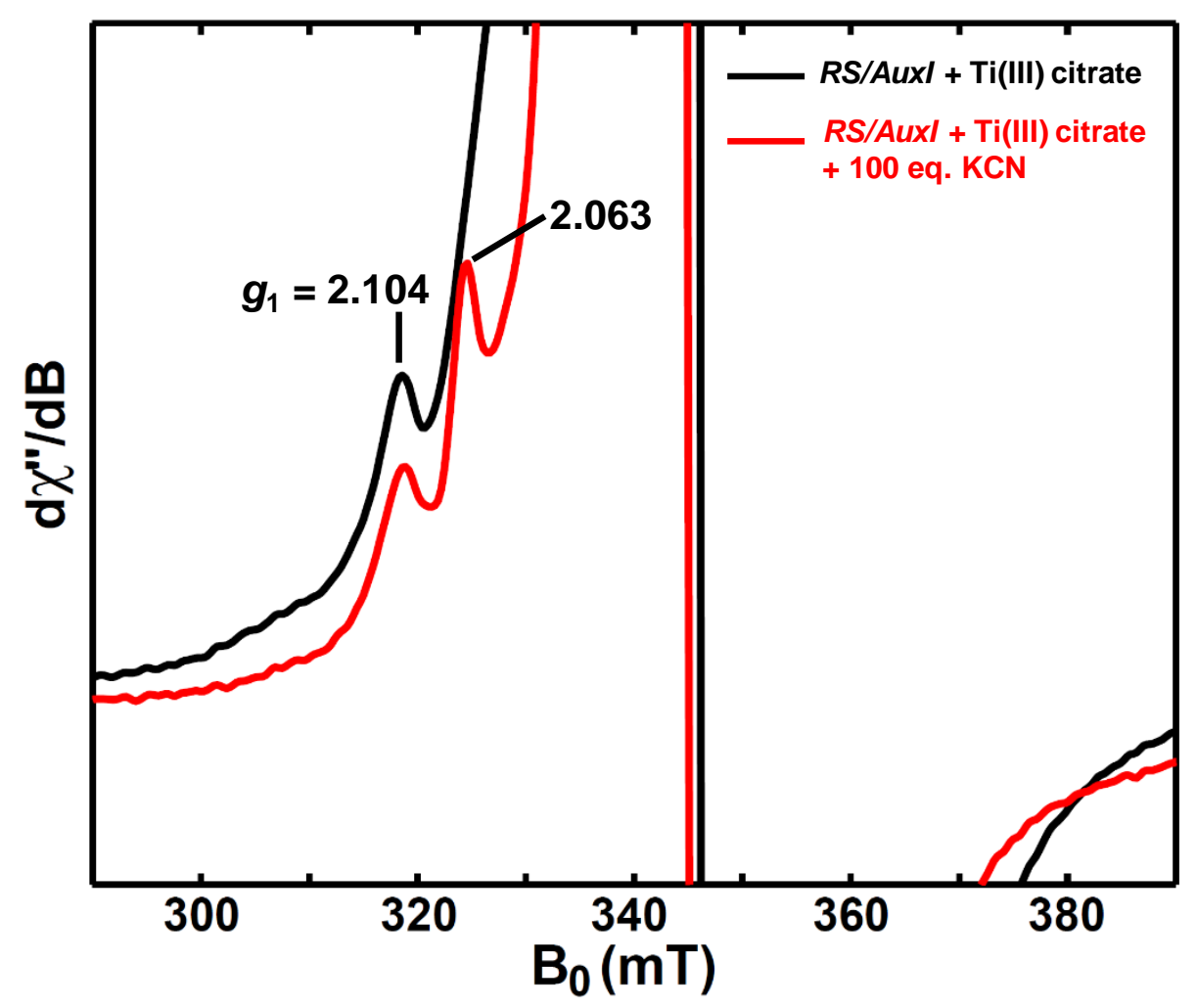

Figure S7. X-band (9.37 GHz) CW EPR spectra of Ti(III) citrate-reduced RS/AuxI PqqE variant (black trace) before (black trace) and after the addition of 110 equivalents of $\mathrm{K}^{13} \mathrm{C}^{15} \mathrm{~N}$. The $\mathrm{CW}$ EPR spectra were recorded at $10 \mathrm{~K}$ using $2.518 \mathrm{~mW}$ microwave power (no saturation). The signal with $g=2.063$ arises from the $\mathrm{CN}$-bound $[4 \mathrm{Fe}-4 \mathrm{~S}]^{+} \mathrm{RS}$ species, as described in section of Characterization of the Radical SAM $[4 \mathrm{Fe}-4 \mathrm{~S}]_{\mathrm{RS}}$ Cluster. 


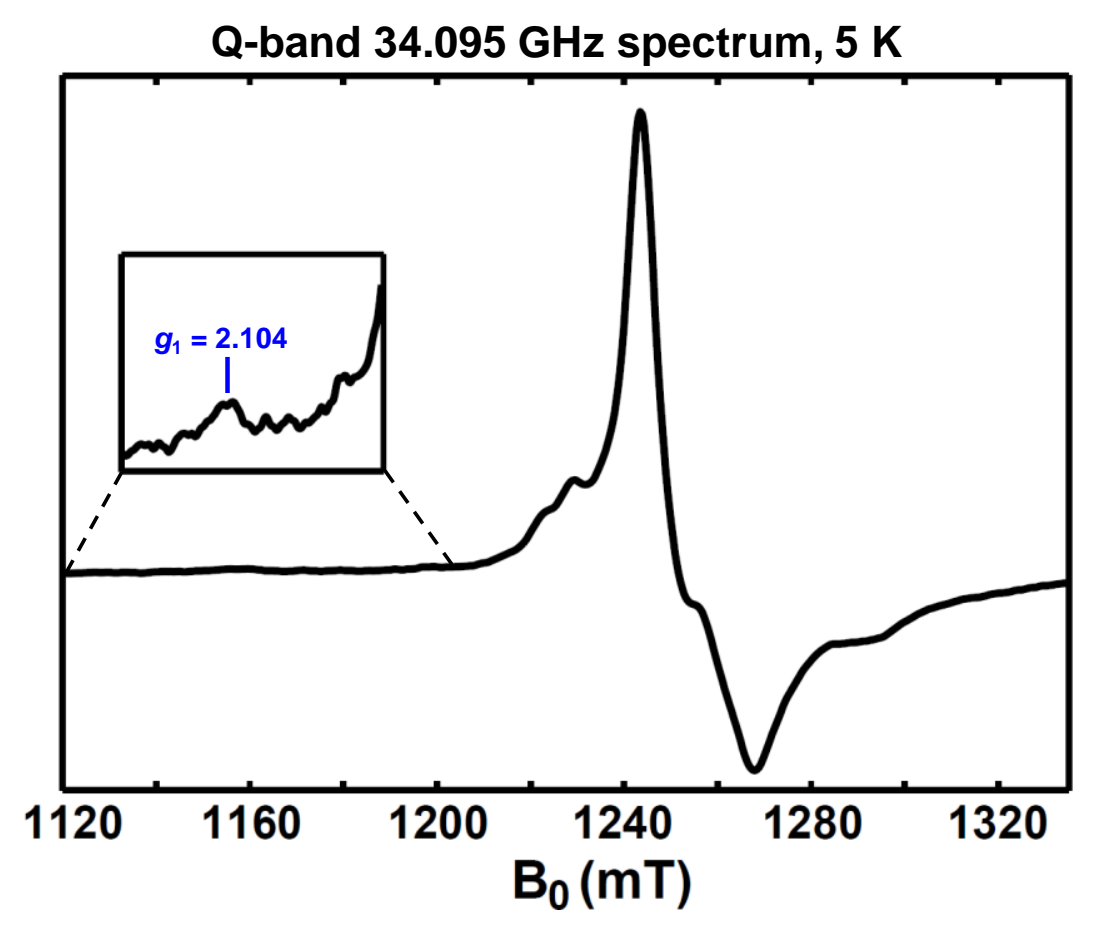

Figure S8. Q-band $(34.0 \mathrm{GHz})$ pseudo-modulated electron spin-echo detected field-swept EPR spectrum of Ti(III) citrate-reduced wild-type PqqE. The spectrum was recorded at $5 \mathrm{~K}$ by using a two-pulse sequence of $\pi / 2-\tau-\pi$ - $\tau$-echo, with $\pi / 2=12 \mathrm{~ns}$ and $\tau=300 \mathrm{~ns}$. The modulation amplitude of $3.0 \mathrm{mT}$ was used to convert the absorption spectrum to the pseudo-modulated spectrum. 

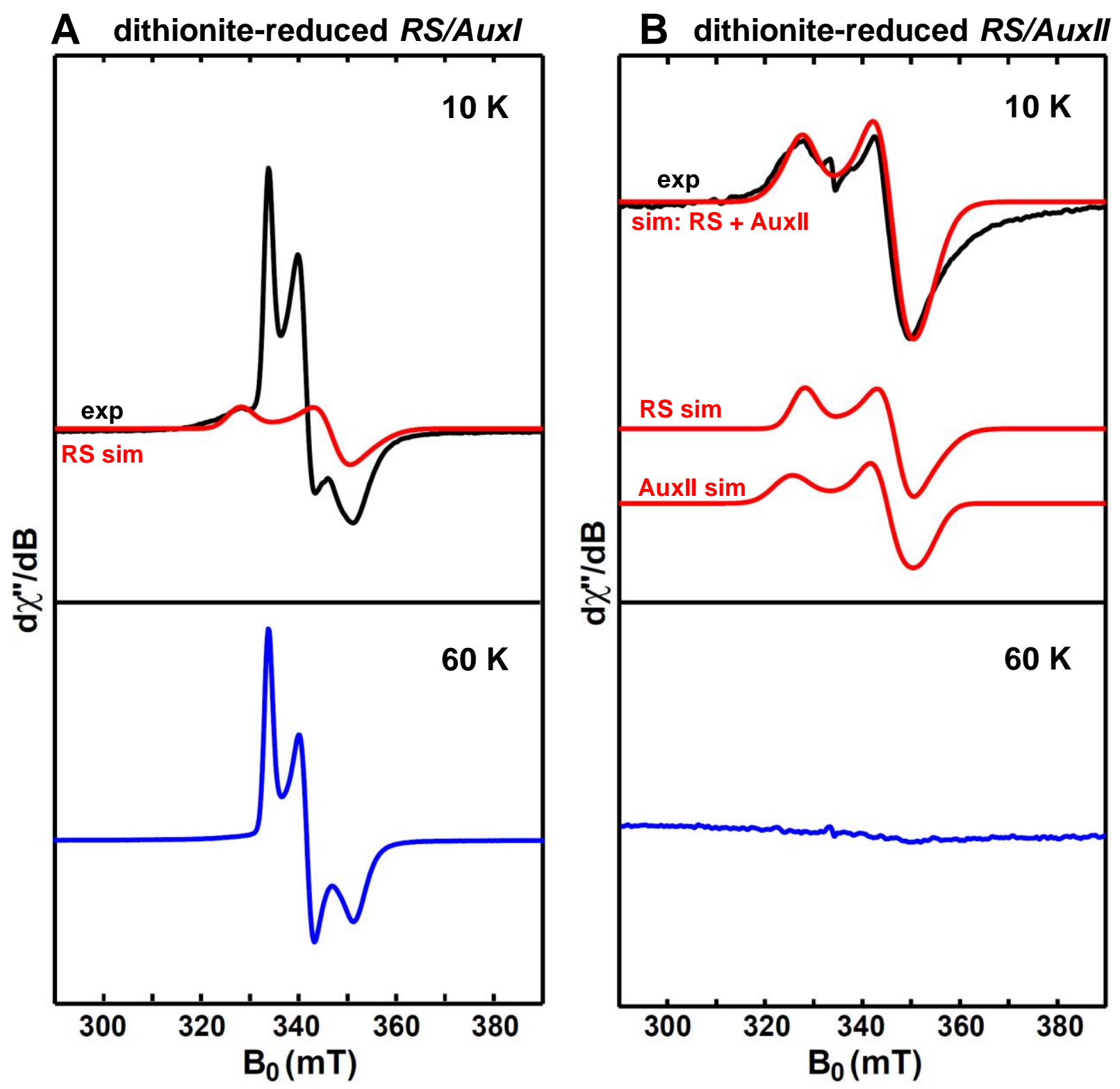

Figure S9. X-band (9.37 GHz) CW EPR spectra of dithionite-reduced RS/AuxI (A) and RS/AuxII (B). The experimental spectrum in (A, black trace) is dominated by the $[2 \mathrm{Fe}-2 \mathrm{~S}]^{+}$cluster signal, as well as the observable component that can be simulated by using the $g$-values $=[2.040,1.927$, 1.897], corresponding to the RS cluster signal.

The experimental spectrum in (B, black trace) has two components. This spectrum can be well simulated by employing two components with the ratio of $1: 1$; one corresponds to the RS cluster with $g$-values $=[2.040,1.927,1.897]$ and the other one is the AuxII cluster with $g$-values $=$ $[2.059,1.940,1.903]$. 

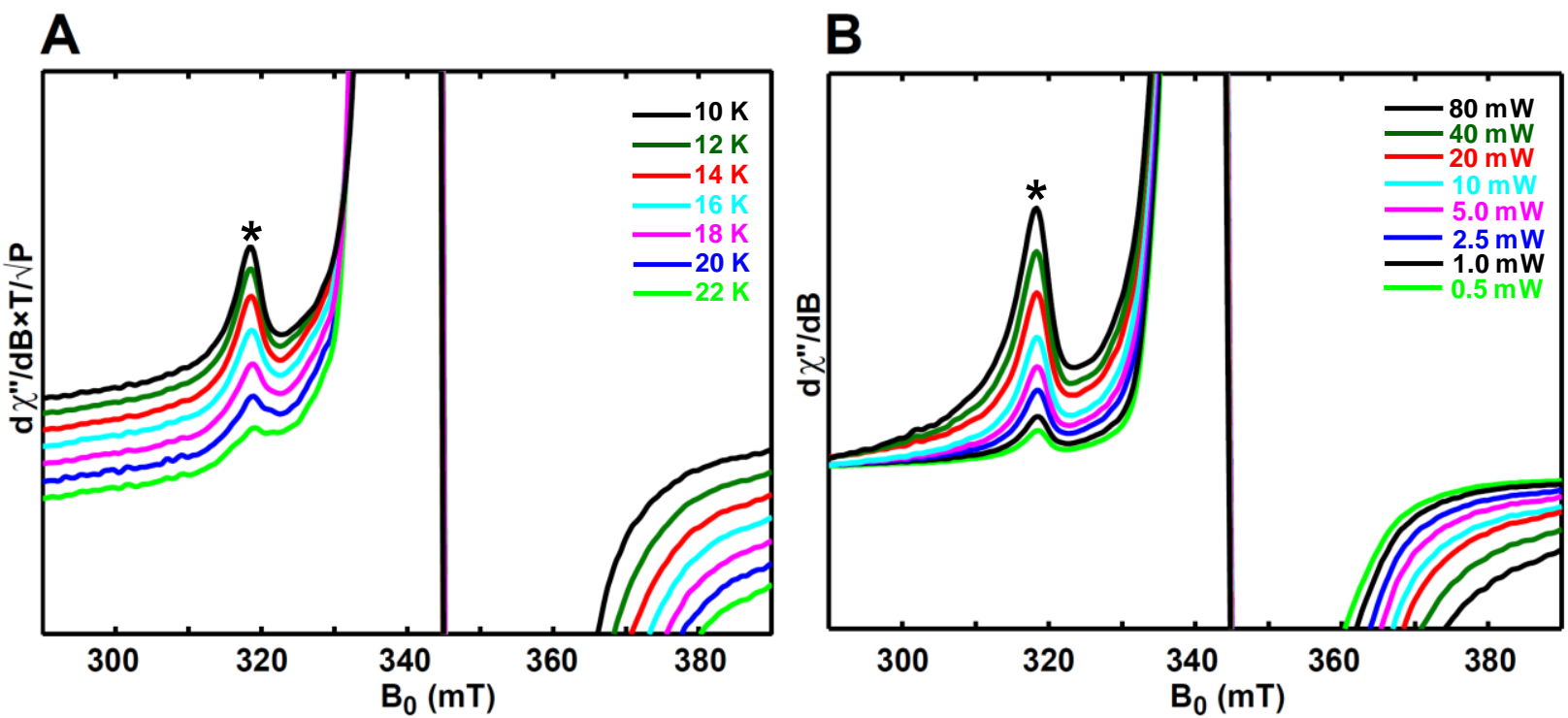

Figure S10. Temperature dependence (A) and power dependence (B) of the EPR signals of Ti(III) citrate-reduced WT PqqE. The asterisk indicates the $g_{1} 2.104$ position, where the peak amplitudes are employed as the signal intensities of the AuxI $[4 \mathrm{Fe}-4 \mathrm{~S}]^{+}$cluster shown in Figure 4 (red circles). 


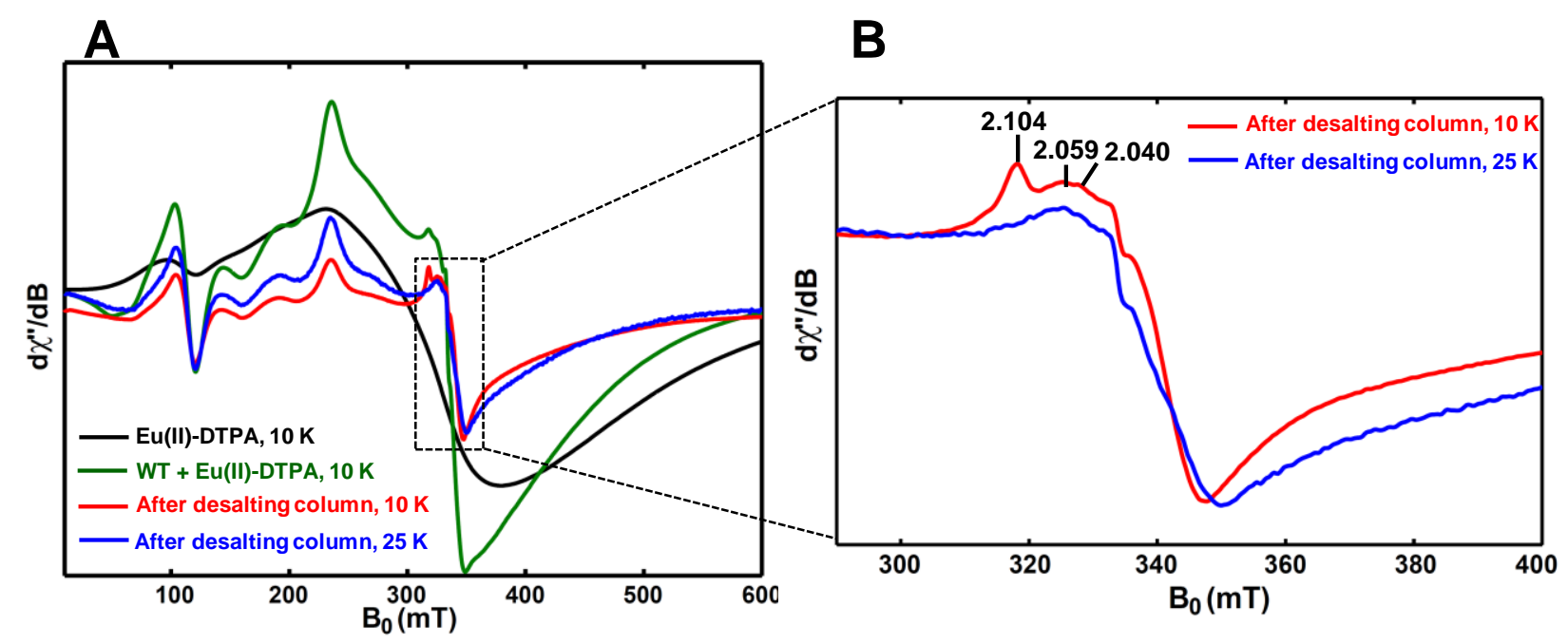

Figure S11. X-band (9.37 GHz) CW EPR spectra of Eu(II)-DTPA-reduced wild-type PqqE before and after desalting. The $g_{1}$ value of 2.104, 2.059 and 2.040 is corresponding to the AuxI $[4 \mathrm{Fe}-4 \mathrm{~S}]^{+}$cluster, the AuxII $[4 \mathrm{Fe}-4 \mathrm{~S}]^{+}$cluster and the RS $[4 \mathrm{Fe}-4 \mathrm{~S}]^{+}$cluster, respectively. The $g_{1} 2.004$ of $[2 \mathrm{Fe}-2 \mathrm{~S}]^{+}$is not well resolved due to the overlap in the central region. The CW EPR spectra were recorded by using $2.518 \mathrm{~mW}$ microwave power at $10 \mathrm{~K}$. 

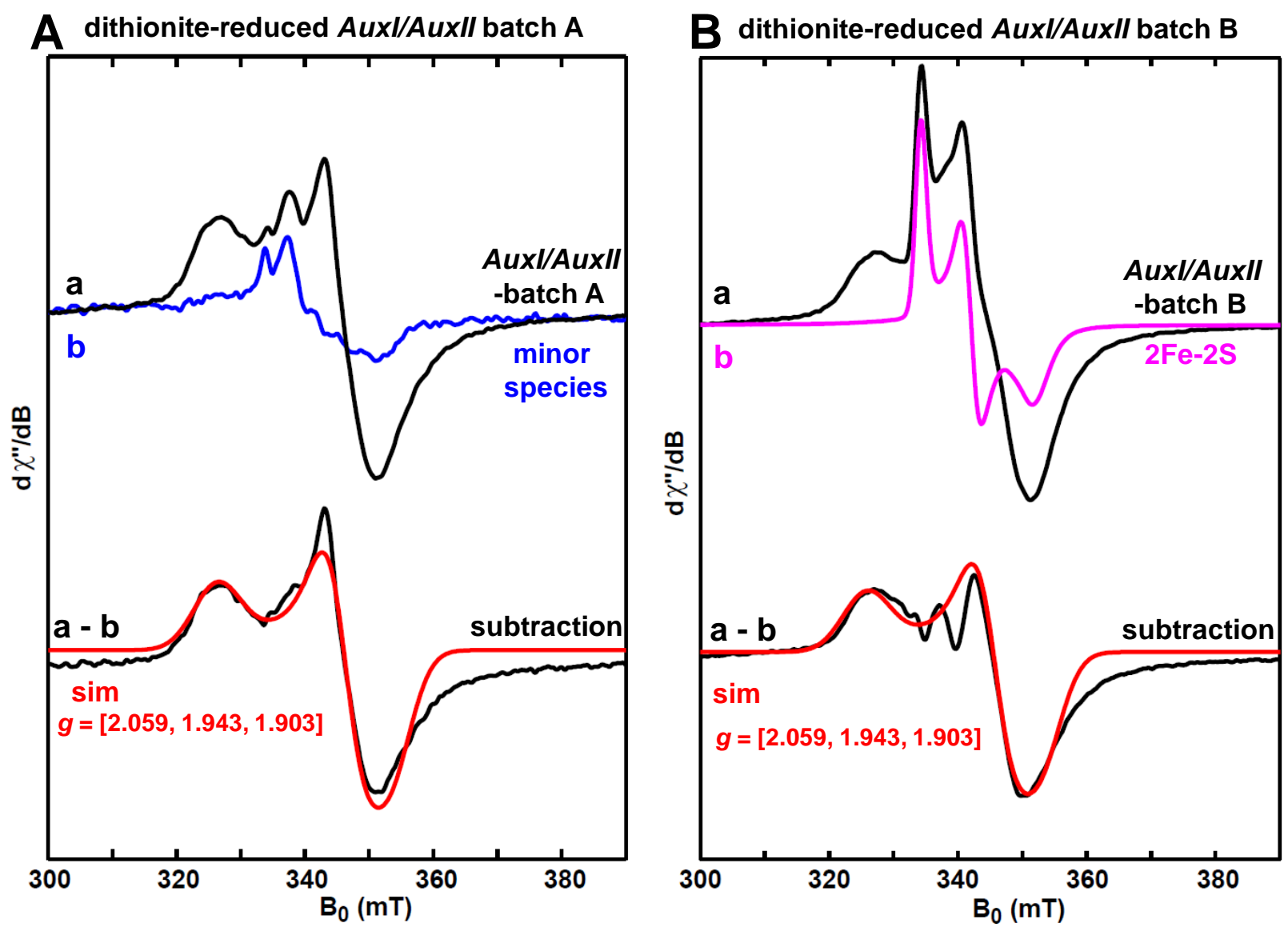

Figure S12. X-band (9.37 GHz) CW EPR of dithionite-reduced two batches of the AuxI/AuxII samples. The batch A spectrum is shown in Figure 6. 

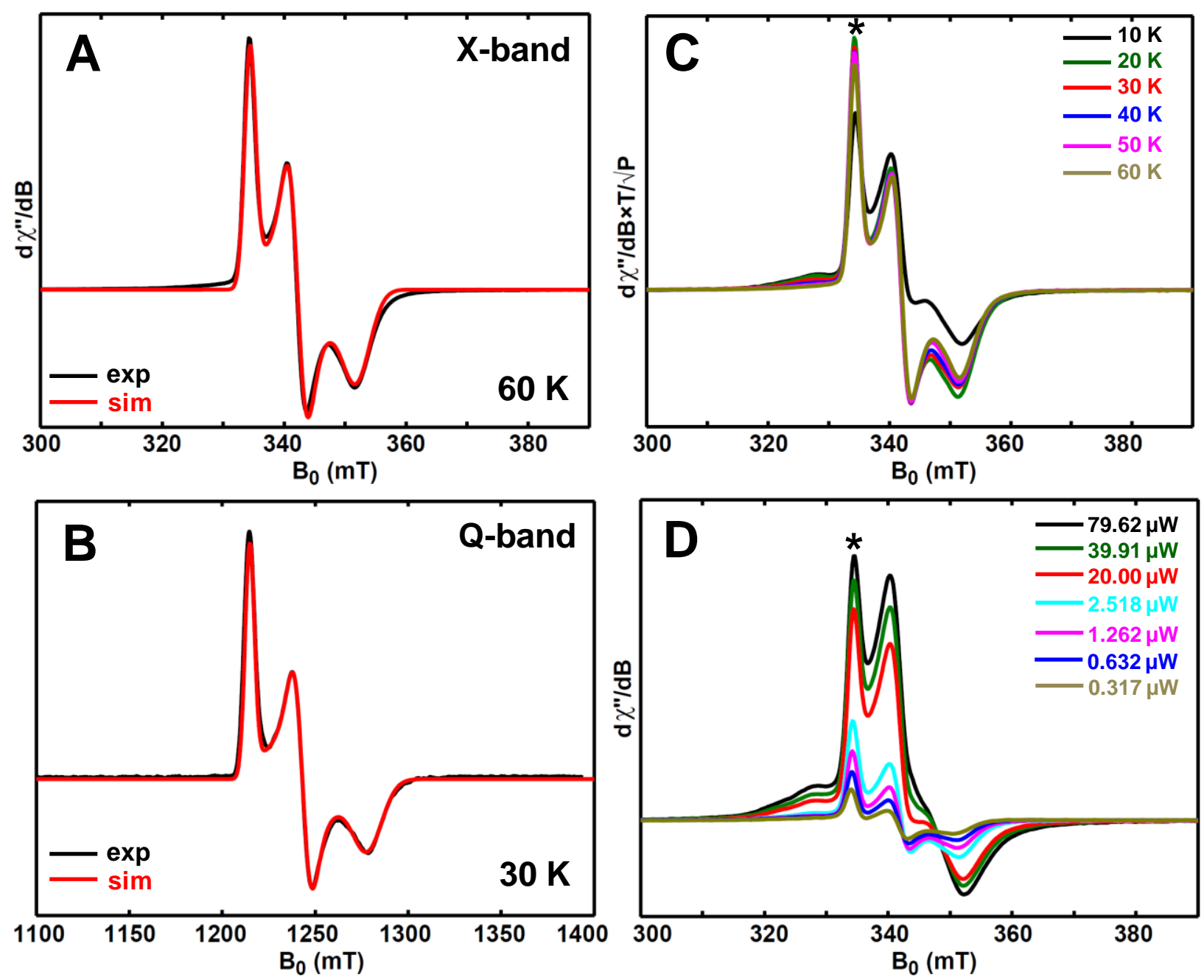

Figure S13. High temperature X-band (9.38 GHz, $60 \mathrm{~K}) \mathrm{CW}$ EPR (A) and Q-band (34.0 GHz, $30 \mathrm{~K}$ ) pseudo-modulated electron spin-echo detected field-swept EPR spectra (B) of dithionitereduced $R S / A u x I$, showing only the $[2 \mathrm{Fe}-2 \mathrm{~S}]^{+}$cluster signals. The black traces are experimental spectra, while the red traces are the simulated spectra by employing the $g$-values $=[2.004,1.958$, 1.904]. The CW EPR spectra were recorded at $60 \mathrm{~K}$, with $0.02 \mathrm{~mW}$ microwave power (no saturation). The Q-band EPR spectra were recorded at $30 \mathrm{~K}$ by using a two-pulse sequence of $\pi / 2-\tau$ - $\pi$ - $\tau$-echo, with $\pi / 2=12 \mathrm{~ns}$ and $\tau=300 \mathrm{~ns}$. The modulation amplitude of $3.0 \mathrm{mT}$ was used to convert the absorption spectra to the pseudo-modulated spectra in (B).

Temperature dependence (C) and power dependence (D) of the EPR signals of dithionitereduced $R S / A u x I$ at $10 \mathrm{~K}$. The asterisk indicates the $g_{1} 2.004$ position, where the peak amplitudes are employed as the signal intensities of the $[2 \mathrm{Fe}-2 \mathrm{~S}]^{+}$cluster (green triangles) shown in Figure 4. The small amount signal intensity from the RS cluster is omitted. 